\title{
Algebraic independence of certain infinite products involving the Fibonacci numbers
}

\author{
By Daniel DuverneY*) and Yohei TACHIYA*) \\ (Communicated by Shigefumi MorI, M.J.A., April 12, 2021)
}

\begin{abstract}
Let $\left\{F_{n}\right\}_{n>0}$ be the Fibonacci sequence. The aim of this paper is to give explicit formulae for the infinite products

$$
\prod_{n=1}^{\infty}\left(1+\frac{1}{F_{n}}\right), \quad \prod_{n=3}^{\infty}\left(1-\frac{1}{F_{n}}\right)
$$

in terms of the values of the Jacobi theta functions. From this we deduce the algebraic independence over $\mathbf{Q}$ of the above numbers by applying Bertrand's theorem on the algebraic independence of the values of the Jacobi theta functions.
\end{abstract}

Key words: Algebraic independence; Fibonacci numbers; Jacobi theta functions.

1. Introduction and main results. Let $\left\{F_{n}\right\}_{n \geq 0}$ be the Fibonacci sequence defined recursively by

$$
F_{n+2}=F_{n+1}+F_{n}, \quad n \geq 0
$$

with $F_{0}=0$ and $F_{1}=1$. Arithmetical properties of infinite sums and products involving the Fibonacci numbers have been investigated by several authors. In 1989, André-Jeannin [1] proved that the sum of the reciprocals of the Fibonacci numbers $\sum_{n=1}^{\infty} 1 / F_{n}$ is irrational (see also $[5,6]$ ). Moreover, it is shown in [7] that the number $\sum_{n=1}^{\infty} 1 / F_{2 n-1}$ is transcendental. On the other hand, some closed forms were discovered in particular cases; for example,

$$
\sum_{n=1}^{\infty} \frac{1}{F_{2^{n}}}=\frac{5-\sqrt{5}}{2}
$$

(cf. [10, p. 225]), which results from the use of telescoping series. As for the infinite products, the second author [12] derived that the numbers

$$
\gamma_{j}:=\prod_{n=1}^{\infty}\left(1+\frac{j}{F_{2^{n}}}\right), \quad j=1,2, \ldots
$$

are all transcendental. The infinite products (2) can be regarded as product analogues of (1). The transcendence result on the $\gamma$ 's was extended in [9] to algebraic independence over $\mathbf{Q}$ of the numbers

2020 Mathematics Subject Classification. Primary 11J85, 11B39, 11F27.

*) Bâtiment A1, 110 rue du chevalier Français, 59000 Lille, France.

**) Graduate School of Science and Technology, Hirosaki University, 1 Bunkyo-cho, Hirosaki, Aomori 036-8561, Japan. $\gamma_{1}, \gamma_{2}, \ldots, \gamma_{m}$ for any integer $m \geq 1$. Also in the case of products, some closed forms can be obtained through the telescoping method. For example, we have

$$
\begin{aligned}
& \prod_{n=1}^{\infty}\left(1+\frac{1}{F_{2 n}}\right)=1+\sqrt{5}, \\
& \prod_{n=2}^{\infty}\left(1-\frac{1}{F_{2 n}}\right)=\frac{1+\sqrt{5}}{6},
\end{aligned}
$$

since

$$
\begin{aligned}
\prod_{n=1}^{\infty}\left(1+\frac{1}{F_{2 n}}\right) & =\prod_{n=1}^{\infty} \frac{\left(1+\alpha^{-2(n-1)}\right)\left(1-\alpha^{-2(n+1)}\right)}{\left(1+\alpha^{-2 n}\right)\left(1-\alpha^{-2 n}\right)} \\
& =\frac{2}{1-\alpha^{-2}}=2 \alpha, \\
\prod_{n=2}^{\infty}\left(1-\frac{1}{F_{2 n}}\right) & =\prod_{n=2}^{\infty} \frac{\left(1+\alpha^{-2(n+1)}\right)\left(1-\alpha^{-2(n-1)}\right)}{\left(1+\alpha^{-2 n}\right)\left(1-\alpha^{-2 n}\right)} \\
& =\frac{1-\alpha^{-2}}{1+\alpha^{-4}}=\frac{\alpha}{3},
\end{aligned}
$$

where $\alpha:=(1+\sqrt{5}) / 2$ is the golden ratio. However, it is not easy to find closed forms and to derive arithmetical properties for given infinite sums and products involving the Fibonacci numbers. For instance, it is not known whether the numbers $\sum_{n=1}^{\infty} 1 / F_{n}$ and $\prod_{n=1}^{\infty}\left(1+1 / F_{3 n}\right)$ are transcendental.

In this paper, we give explicit formulae for the two fundamental infinite products

$$
\prod_{n=1}^{\infty}\left(1+\frac{1}{F_{n}}\right)=13.1509666577 \ldots
$$




$$
\prod_{n=3}^{\infty}\left(1-\frac{1}{F_{n}}\right)=0.1897891436 \ldots
$$

by means of the values of the Jacobi theta functions. Moreover, by using the formulae we prove that the above two numbers are algebraically independent over $\mathbf{Q}$. To state our results, we define the Jacobi theta functions

$$
\left\{\begin{array}{l}
\vartheta_{2}(q):=2 q^{1 / 4} \sum_{n=0}^{\infty} q^{n(n+1)}, \\
\vartheta_{3}(q):=1+2 \sum_{n=1}^{\infty} q^{n^{2}}, \\
\vartheta_{4}(q):=1+2 \sum_{n=1}^{\infty}(-1)^{n} q^{n^{2}},
\end{array}\right.
$$

which converge for all complex numbers $q$ with $|q|<1$. Throughout this paper, let

$$
\beta:=\frac{1}{\alpha}=\frac{\sqrt{5}-1}{2} .
$$

Our main results are the following

Theorem 1. Let $\left\{F_{n}\right\}_{n \geq 0}$ be the Fibonacci sequence. Then

$$
\begin{gathered}
\xi_{1}:=\prod_{n=1}^{\infty}\left(1+\frac{1}{F_{n}}\right)=2 \beta^{-5 / 4} \frac{\vartheta_{2}(\beta)}{\vartheta_{4}\left(\beta^{4}\right)} \\
\xi_{2}:=\prod_{n=3}^{\infty}\left(1-\frac{1}{F_{n}}\right)=\frac{\sqrt{5}}{6} \beta^{-5 / 4} \frac{\vartheta_{2}(\beta) \vartheta_{3}(\beta) \vartheta_{4}(\beta)}{\vartheta_{4}\left(\beta^{4}\right)} .
\end{gathered}
$$

Theorem 2. The numbers $\xi_{1}$ and $\xi_{2}$ are algebraically independent over $\mathbf{Q}$. In particular, the numbers $\xi_{1}$ and $\xi_{2}$ are both transcendental.

2. Proofs of Theorems 1 and 2. We first prove Theorem 1. Let $\vartheta_{2}(q), \vartheta_{3}(q), \vartheta_{4}(q)$ be the Jacobi theta functions defined in (5). Using the triple-product identities

$$
\begin{aligned}
& \vartheta_{2}(q)=q^{1 / 4} \prod_{n=1}^{\infty}\left(1-q^{2 n}\right)\left(1+q^{2 n-2}\right)\left(1+q^{2 n}\right), \\
& \vartheta_{3}(q)=\prod_{n=1}^{\infty}\left(1-q^{2 n}\right)\left(1+q^{2 n-1}\right)^{2}, \\
& \vartheta_{4}(q)=\prod_{n=1}^{\infty}\left(1-q^{2 n}\right)\left(1-q^{2 n-1}\right)^{2}
\end{aligned}
$$

(cf. [4, Corollary 3.1]), we have

$$
q^{-1 / 4} \vartheta_{2}(q)=\prod_{n=1}^{\infty}\left(1-q^{2 n}\right)\left(1+q^{2 n-2}\right)\left(1+q^{2 n}\right)
$$

$$
\begin{gathered}
\vartheta_{2}(q) \vartheta_{3}(q) \vartheta_{4}(q)=2 q^{1 / 4} \prod_{n=1}^{\infty}\left(1-q^{2 n}\right)^{3} \\
\vartheta_{4}\left(q^{4}\right)=\prod_{n=1}^{\infty}\left(1-q^{4 \cdot 2 n}\right)\left(1-q^{4(2 n-1)}\right)^{2} \\
=\prod_{n=1}^{\infty}\left(1-q^{4 n}\right)\left(1-q^{4(2 n-1)}\right) \\
=\prod_{n=1}^{\infty}\left(1-q^{2 n}\right)\left(1+q^{4 n-2}\right)
\end{gathered}
$$

(see [4, Proof of Proposition 3.1] for (8)). Hence, we obtain by $(7),(8)$, and $(9)$

$$
\begin{aligned}
\prod_{n=1}^{\infty}\left(1+\frac{1}{F_{2 n-1}}\right) & =\prod_{n=1}^{\infty} \frac{\left(1+\beta^{2 n-2}\right)\left(1+\beta^{2 n}\right)}{1+\beta^{4 n-2}} \\
& =\beta^{-1 / 4} \frac{\vartheta_{2}(\beta)}{\vartheta_{4}\left(\beta^{4}\right)} \\
\prod_{n=2}^{\infty}\left(1-\frac{1}{F_{2 n-1}}\right) & =\prod_{n=2}^{\infty} \frac{\left(1-\beta^{2 n-2}\right)\left(1-\beta^{2 n}\right)}{1+\beta^{4 n-2}} \\
& =\frac{\beta^{-1 / 4}\left(1+\beta^{2}\right)}{2\left(1-\beta^{2}\right)} \frac{\vartheta_{2}(\beta) \vartheta_{3}(\beta) \vartheta_{4}(\beta)}{\vartheta_{4}\left(\beta^{4}\right)} .
\end{aligned}
$$

Therefore, Theorem 1 follows from the above formulae and (3), (4), (6).

Next we show Theorem 2. Using the wellknown identities

$$
\begin{aligned}
\vartheta_{2}^{2}(q) & =2 \vartheta_{2}\left(q^{2}\right) \vartheta_{3}\left(q^{2}\right), \\
\vartheta_{4}^{2}\left(q^{2}\right) & =\vartheta_{3}(q) \vartheta_{4}(q),
\end{aligned}
$$

(cf. [4, Chapter 2, §2.1]), we obtain by Theorem 1

$$
\begin{aligned}
\xi_{1}^{3} \xi_{2} & =\frac{16 \sqrt{5}}{3} \beta^{-5} \vartheta_{2}^{2}\left(\beta^{2}\right), \\
\frac{\xi_{2}}{\xi_{1}} & =\frac{\sqrt{5}}{12} \vartheta_{4}^{2}\left(\beta^{2}\right),
\end{aligned}
$$

so that the numbers $\vartheta_{2}^{2}\left(\beta^{2}\right)$ and $\vartheta_{4}^{2}\left(\beta^{2}\right)$ belong to the field $\mathbf{Q}\left(\xi_{1}, \xi_{2}, \beta\right)$. Hence, putting $\mathbf{K}:=\mathbf{Q}\left(\xi_{1}, \xi_{2}\right)$ and noting that $\beta$ is an algebraic number, we have

$$
\begin{aligned}
2 & \geq \text { trans.deg } \operatorname{de}_{\mathbf{Q}} \mathbf{K}=\text { trans.deg } \mathbf{Q} \mathbf{Q}\left(\xi_{1}, \xi_{2}, \beta\right) \\
& \geq \text { trans.deg } \mathbf{Q} \mathbf{Q}\left(\vartheta_{2}^{2}\left(\beta^{2}\right), \vartheta_{4}^{2}\left(\beta^{2}\right)\right)=2,
\end{aligned}
$$

where at the last equality we used a result of Bertrand [3, Theorem 4] (see also [8]) which is a consequence of Nesterenko's theorem [11] on the algebraic independence of the values of Ramanujan functions. This implies that trans.deg ${ }_{\mathbf{Q}} \mathbf{K}=2$, namely, the numbers $\xi_{1}$ and $\xi_{2}$ are algebraically 
independent over $\mathbf{Q}$. The proof of Theorem 2 is completed.

Remark 1. It should be noted that the transcendence of $\xi_{1}$ is an immediate consequence of a result of Barré-Sirieix, Diaz, Gramain and Philibert [2] (see also [3, Theorem 3]). Indeed, by Theorem 1 and the identities (10) and (11), we have

$$
\xi_{1}^{2}=8 \beta^{-5 / 2} \frac{\vartheta_{2}\left(\beta^{2}\right)}{\vartheta_{4}\left(\beta^{2}\right)}
$$

Thus, we find by $[2$, Théorème (i)] that the number $\vartheta_{2}\left(\beta^{2}\right) / \vartheta_{4}\left(\beta^{2}\right)$ is transcendental, and hence, so is $\xi_{1}$.

Acknowledgments. The authors wish to express their sincere gratitude to the referee for valuable comments. This work was supported by JSPS KAKENHI Grant Number JP18K03201.

\section{References}

[ 1 ] R. André-Jeannin, Irrationalité de la somme des inverses de certaines suites récurrentes, C. R. Acad. Sci. Paris Sér. I Math. 308 (1989), no. 19, 539-541.

[ 2 ] K. Barré-Sirieix, G. Diaz, F. Gramain and G. Philibert, Une preuve de la conjecture de Mahler-Manin, Invent. Math. 124 (1996), no. 1-3, 1-9.

[ 3 ] D. Bertrand, Theta functions and transcendence, Ramanujan J. 1 (1997), no. 4, 339-350.
[ 4 ] J. M. Borwein and P. B. Borwein, Pi and the $A G M$, reprint of the 1987 original, Canadian Mathematical Society Series of Monographs and Advanced Texts, 4, John Wiley \& Sons, Inc., New York, 1998.

[ 5 ] P. Bundschuh and K. Väänänen, Arithmetical investigations of a certain infinite product, Compositio Math. 91 (1994), no. 2, 175-199.

[ 6 ] D. Duverney, Irrationalité de la somme des inverses de la suite de Fibonacci, Elem. Math. 52 (1997), no. 1, 31-36.

[ 7 ] D. Duverney, Ke. Nishioka, Ku. Nishioka and I. Shiokawa, Transcendence of Jacobi's theta series and related results, in Number theory (Eger, 1996), 157-168, de Gruyter, Berlin, 1998.

[ 8 ] C. Elsner, M. Kaneko and Y. Tachiya, Algebraic independence results for the values of the thetaconstants and some identities, J. Ramanujan Math. Soc. 35 (2020), no. 1, 71-80.

[ 9 ] T. Kurosawa, Y. Tachiya and T. Tanaka, Algebraic independence of infinite products generated by Fibonacci numbers, Tsukuba J. Math. 34 (2010), no. 2, 255-264.

[ 10 ] E. Lucas, Théorie des fonctions numériques simplement périodiques, Amer. J. Math. 1 (1878), nos. 2-3, 184-240.

[11] Yu. V. Nesterenko, Modular functions and transcendence questions, Mat. Sb. 187 (1996), no. 9, 65-96; English transl. Sb. Math. 187 (1996), no. 9, 1319-1348.

[12 ] Y. Tachiya, Transcendence of certain infinite products, J. Number Theory 125 (2007), no. $1,182-200$. 